\title{
Multiobjective Transmission Network Planning considering the Uncertainty and Correlation of Wind Power
}

\author{
Yuan Hu, ${ }^{1}$ Zhaohong Bie, ${ }^{1}$ Yanling Lin, ${ }^{1}$ Guangtao Ning, ${ }^{2}$ Mingfan Chen, ${ }^{2}$ and Yujie Gao ${ }^{2}$ \\ ${ }^{1}$ State Key Laboratory of Electrical Insulation and Power Equipment, School of Electrical Engineering, \\ Xian Jiaotong University, Xian 710049, China \\ ${ }^{2}$ Hainan Power Grid Corporation, Haikou 570203, China
}

Correspondence should be addressed to Zhaohong Bie; zhbie@mail.xjtu.edu.cn

Received 24 January 2014; Accepted 5 June 2014; Published 1 July 2014

Academic Editor: H. D. Chiang

Copyright (C) 2014 Yuan Hu et al. This is an open access article distributed under the Creative Commons Attribution License, which permits unrestricted use, distribution, and reproduction in any medium, provided the original work is properly cited.

\begin{abstract}
In order to consider the uncertainty and correlation of wind power in multiobjective transmission network expansion planning (TNEP), this paper presents an extended point-estimation method to calculate the probabilistic power flow, based on which the correlative power outputs of wind farm are sampled and the uncertain multiobjective transmission network planning model is transformed into a solvable deterministic model. A modified epsilon multiobjective evolutionary algorithm is used to solve the above model and a well-distributed Pareto front is achieved, and then the final planning scheme can be obtained from the set of nondominated solutions by a fuzzy satisfied method. The proposed method only needs the first four statistical moments and correlation coefficients of the output power of wind farms as input information; the modeling of wind power is more precise by considering the correlation between wind farms, and it can be easily combined with the multiobjective transmission network planning model. Besides, as the self-adaptive probabilities of crossover and mutation are adopted, the global search capabilities of the proposed algorithm can be significantly improved while the probability of being stuck in the local optimum is effectively reduced. The accuracy and efficiency of the proposed method are validated by IEEE 24 as well as a real system.
\end{abstract}

\section{Introduction}

With the increasing complexity of power system, transmission network expansion planning needs to consider multiple objectives, such as investment cost, security, and network losses. Since these objectives may be conflicting with each other, it is hard for the a priori approaches, such as weighted method and goal programming [1-3], to reach a good trade-off between different objectives and find the global optimal plan. With the advantages of handling incommensurable objectives with conflicting relations and the powerful global search abilities, multiobjective evolutionary algorithms (MOEAs), such as SPEA [4], NSGA2 [5], are gaining increasing popularity among researchers and practitioners [6] and have been widely used in the TNEP [7-10]. MOEA is an iterative search algorithm, and the computational time to achieve the final result is closely related to the complexity of evaluation of objective function. Because TNEP is a nonlinear, nonconvex, and mixed integer optimization problem, and if the uncertainty of power system is also considered, it can be very difficult to solve; thus, a suitable method to handle uncertainty is also necessary in the multiobjective TNEP.

There are many uncertain factors affecting the TNEP, and one of the current research focuses is wind power [11, 12]. With the benefits of assessing network operation over a variety of working conditions in a computationally efficient manner, probabilistic power flow (PPF) [13] that is based on different techniques has been widely applied to model the intermittency and volatility of wind power in TNEP $[9,14-$ 17]. These techniques can be classified into three main categories [18]: Monte Carlo simulation (MCS) [15, 19], analytical methods [14], and approximate methods [9]. MCS can reach high accuracy, but it requires a large number of simulations to attain convergence. Analytic methods are computationally effective; however, it requires the linear relationship between the output variable and input random variable, which is not consistent with the actual situation of power system 
[20]. Point-estimate method as an approximate method was proposed by Hong in 1998 [21]. Since this method not only requires less information on input data but also can achieve a balance between the accuracy of the results and the efficiency of the computational procedure, it provides an effective tool to calculate the PPF and makes the combination of MOEA and PPF solved by point-estimate method possible.

Although much work has been done to handle the influence of wind power in TNEP, the correlation between wind farms is seldom considered [12]. In real operation, the outputs of wind farms that are distributed geographically close to each other may increase or decrease at the same time, and the effect of such correlation between wind farms cannot be neglected in the power flow on the network, so it is imperative to consider the correlation between wind farms when modeling the wind power in TNEP $[8,9]$.

In this paper, we establish a multiobjective TNEP model considering the investment cost and network losses cost as well as the sum of the line overload capacity both under the normal operation and single contingency condition. Based on the proposed model, an extended $2 m+1$ point-estimate method is used to deal with the uncertainty and correlation of wind power. With the same computational process as in the associated deterministic problems, and only requiring $2 m+1$ calculation ( $m$ represents the number of variables), this method has low computational burden and can easily be combined with the multiobjective TNEP model. Besides, only the first four statistical moments of wind power are required as input information, and the correlation between wind farms is considered; the modeling of wind power is more simple and accurate. After transforming the uncertain multiobjective model into a solvable deterministic model, a modified $\varepsilon$-MOEA is applied to obtain the final optimalPareto front. Since probabilities of crossover and mutation in the proposed algorithm are updated in each iteration, depending on the fitness value of population, the global search capability is strengthened and the probability of being stuck in the local optimum is reduced. Finally, the final plan can be achieved from the set of nondominated solutions by a fuzzy satisfying method.

The rest of the paper is organized as follows. After the wind power is modeled, an extended $2 m+1$ point-estimate method is described in Section 2. In Section 3, the mathematical formulation of the TNEP and a decision making method are introduced, followed by Section 4 presenting the proposed multiobjective optimization algorithm. Section 5 is devoted to show the capabilities of the proposed method by case study. Finally, conclusions are given in Section 6.

\section{Modeling}

2.1. Wind Model Based on the $2 m+1$ Point-Estimate Method. In the previous work, Weibull distribution is widely used to sample the wind speed $[22,23]$, and the parameters of the distribution can be derived from the mean and standard deviation of historical wind speed [24]. The relationship between the available power of wind turbine generator and the wind speed is commonly characterized by the following function:

$$
P_{w}= \begin{cases}0 & V<V_{\mathrm{ci}}, V>V_{\text {co }} \\ \frac{P_{\text {rate }}\left(V-V_{\mathrm{ci}}\right)}{\left(V_{\text {rate }}-V_{\mathrm{ci}}\right)} & V_{\mathrm{ci}} \leq V<V_{\text {rate }} \\ P_{\text {rate }} & V_{\text {rate }} \leq V \leq V_{\text {co }}\end{cases}
$$

where $P_{\text {rate }}$ is the rated power of wind turbine, $V_{\mathrm{ci}}, V_{\text {rate }}$, and $V_{\text {co }}$ are the cut-in, rated, cut-out wind speed, respectively.

The formulation above is a piecewise function, so it is hard to obtain the distribution of the wind turbine output. In this paper, we adopt the yearly historical outputs of wind farms as input data, and the distribution of wind farm output is described by its first four statistical moments, including the mean value, standard deviation, skewness, and kurtosis, in which the skewness is a measure of the extent to which a probability distribution of a real-valued random variable "leans" to one side of the mean, and the kurtosis is a measure of both the "peakedness" of the distribution and the heaviness of its tail. These four statistics can be obtained from the historical wind power data easily; given the historical wind speed data, the output power of wind farm can be achieved by (1).

In order to improve the accuracy of the modeling of wind power generation by considering the spatial correlation among the adjacent wind farms, here we use the variancecovariance matrix, which can be easily achieved from the yearly curve of output from wind farms, to characterize such correlation between wind farms.

\subsection{Probabilistic Power Flow Solved by $2 m+1$ Point-Estimate} Method considering the Correlation of Input Data. Basic theory of point-estimate method is introduced in [21]. The procedure of using point-estimate method to solve PPF is as follows: firstly, concentrate the statistical information of the input random variable on $k$ points and weights for each variable by its first few central moments, where $k$ is determined by the user; then use these points to calculate the deterministic power flow; finally, the statistical information of output random variables can be obtained by the weight sum of each deterministic result. Since relatively accurate results can be achieved while keeping the low computational burden, PPF solved by point-estimate method was widely used to handle uncertain factors in power system now [9, 25, 26]. Among different point-estimate schemes proposed by Hong [21], $2 m+1$ point-estimate method shows the best performance on solving probabilistic power flow problem [18].

Although most of the input random variables in power system are statistically dependent, the previous work, however, has sidestepped it. To consider dependencies among the input random variables when calculating the PPF, an orthogonal transformation is used to transform the dependent input random variables into the independent ones, and then it can be processed readily through $2 m+1$ point-estimate methods. PPF solved by the extended point-estimate method [27] is as follows. 
(1) Input the first four statistical moments of the $m$ input random variables $\left(X=\left[X_{1}, X_{2}, \ldots, X_{m}\right]\right)$, including the means $\mu_{X}$, standard deviations $\delta_{X}$, skewness $\lambda_{X, 3}$ and kurtosis $\lambda_{X, 4}$, as well as the variance-covariance matrix $C_{X}$.

(2) Decompose $C_{X}$ by Cholesky decomposition to obtain an inferior triangular matrix $L, C_{X}=L L^{T}$.

(3) Set the iteration count $l=1$, Initialize $E(Y)^{(1)}=0$, $Y=H(X)$.

(4) Transform the first four central moments of the input variables as follows:

$$
\begin{gathered}
\mu_{Z}=L^{-1} \mu_{X}, \\
C_{Z}=L^{-1} C_{X}\left(L^{-1}\right)^{T}=I, \\
\lambda_{Z l, 3}=\sum_{r=1}^{m}\left(L_{l r}^{-1}\right)^{3} \lambda_{X r, 3} \delta_{X r}, \\
\lambda_{Z l, 4}=\sum_{r=1}^{m}\left(L_{l r}^{-1}\right)^{4} \lambda_{X r, 4} \delta_{X r} .
\end{gathered}
$$

(5) Calculate the concentrations $\left(\left(Z_{l, k}, \omega_{l, k}\right), k=1,2,3\right)$ in the transformed space according to the following equation:

$$
\begin{gathered}
\xi_{l, k}=\frac{\lambda_{Z l, 3}}{2}+(-1)^{3-k} \sqrt{\lambda_{Z l, 4}-\frac{3}{4} \lambda_{Z l, 3}^{2}}, \quad k=1,2, \\
\xi_{l, 3}=0, \\
\omega_{l, k}=\frac{(-1)^{3-k}}{\xi_{l, k}\left(\xi_{l, 1}-\xi_{l, 2}\right)}, \quad k=1,2, \\
\omega_{l, 3}=\frac{1}{m}-\frac{1}{\lambda_{Z l, 4}-\lambda_{Z l, 3}^{2}}, \\
Z_{l, k}=\mu_{Z l}+\xi_{l, k} \cdot \delta_{Z l}, \quad k=1,2 .
\end{gathered}
$$

(6) Construct the transformed points in the form $\left(Z_{l}^{k}=\right.$ $\left.\left(\mu_{Z 1}, \mu_{Z 2}, \ldots Z_{l, k}, \ldots, \mu_{Z m}\right), k=1,2,3\right)$, and convert the points into the original space by applying the inverse transformation; that is, $X_{l}^{k}=L Z_{l}^{k}, k=1,2,3$.

(7) Solve a deterministic DC power flow for each of the three points $\left(Y_{l}^{k}=H\left(X_{l}^{k}\right), k=1,2,3\right)$, updating the following equation:

$$
E(Y)^{(l+1)} \cong E(Y)^{(l)}+\sum_{k=1}^{3}\left(\omega_{l, k} \times H\left(X_{l}^{k}\right)\right)
$$

(8) Since $m$ of $3 m$ points are at the same point $\left(\mu_{X 1}, \mu_{X 2}, \ldots \mu_{X k}, \ldots, \mu_{X m}\right)$, so after $2 m+1$ iterations, approximation of the mean value of $Y$ is $\mu_{Y} \cong$ $\sum_{l=1}^{m} \sum_{k=1}^{3} \omega_{l, k} \times H\left(X_{l}^{k}\right)$.

\section{Mathematical Formulation}

3.1. Transmission Planning Formulation. Economic and security criteria are the most important factors in the transmission expansion planning. Based on [28], in this paper, the investment cost and network losses cost as well as the sum of the line overload capacity both under the $\mathrm{N}$ security check and " $\mathrm{N}$ 1 " security check are chosen as the objective functions. The proposed multiobjective programming model is as follows.

\subsubsection{Objective Functions}

(a) Investment Cost. Consider.

$$
\min f_{1}=k(r, n) \sum_{l \in \Omega} C_{l} x_{l}
$$

where $C_{l}$ is the cost of the added line $l$ and $x_{l}$ is the binary decision variable, in which " 0 " represents the candidate line is not added, while " 1 " represents the candidate line is added. $\Omega$ is the set of candidate lines. $k(r, n)$ is funds coefficient of recovery calculated by

$$
k(r, n)=\frac{r(1+r)^{n}}{\left((1+r)^{n}-1\right)},
$$

where $r$ is the discount rate and $n$ is the year limit of discount.

(b) Amount of Line Overload Capacity. Consider.

$$
\begin{gathered}
\min f_{2}=\beta_{1} \sum_{k \in \Gamma} \text { Overload }_{k}+\sum_{m n \in \Psi} \sum_{k \in \Gamma} \text { Overload }_{k}^{m n}, \\
\text { Overload }_{k}= \begin{cases}0 & \left|P_{k}\right| \leq \overline{P_{k}} \\
\left|P_{k}\right|-P_{k} & \left|P_{k}\right|>\overline{P_{k}},\end{cases}
\end{gathered}
$$

where $P_{k}$ is the active power flow of line $k, \overline{P_{k}}$ is the maximum capacity of line $k$, and Overload $_{k}$ and $\operatorname{Overload}_{k}^{m n}$ are the overload capacity of line $k$ under normal operation without contingencies and single contingency condition in which line $m n$ is out of service, respectively. $\Gamma$ is the set of overload lines; $\Psi$ is the set of selected contingencies. $\beta_{1}$ is the penalty coefficient. Generally, to guarantee that the final solutions have no load curtailment in normal operation, the value of $\beta_{1}$ should be large enough.

(c) The Cost of Network Losses. Consider.

$$
\min f_{3}=T \sum_{k \in \Omega(0)} C_{e} R_{k} P_{k}^{2}
$$

where $C_{e}$ is unit electricity price, $R_{k}$ is resistance of line $k, P_{k}$ is the active power flow of line $k$ in normal operation, $T$ is the annual network losses hour, and $\Omega(0)$ is the set of both existing and candidate lines.

3.1.2. Constraints. The constraints of the above multiobjective optimization problem are as follows. 
Network under normal conditions:

$$
\begin{gathered}
B \theta=P_{G}-P_{D}, \\
B_{l} A \theta=P_{k}, \\
\left|P_{k}\right| \leq \overline{P_{k}} .
\end{gathered}
$$

Network under single contingency conditions:

$$
\begin{gathered}
B^{m n} \theta^{m n}=P_{G}-P_{D}, \\
B_{l}^{m n} A^{m n} \theta^{m n}=P_{k}^{m n}, \\
\left|P_{k}^{m n}\right| \leq \overline{P_{k}},
\end{gathered}
$$

where $P_{G}$ is the column vector of generated active powers, $P_{D}$ is the column vector of predicted loads, $B$ is the nodal admittance matrix, $B_{l}$ is the diagonal matrix formed by susceptances of all the network lines, $A$ is the branch-node incidence matrix, and $\theta$ is the phase angle column vector of nodal voltage. Superscript $m n$ denotes the parameters or variables under the single contingency after outage of line $m n$.

In the above constraints, the constraint (10) represents the active power balance at node and the operational limit of each transmission line under normal operational conditions. In single line contingency (e.g., when the line is cut off), the constraint (10) is transformed into the constraint (11) to guarantee the single contingency security or N-1 security. For objective function (7), both constraints (10) and (11) are active; when minimizing the cost of network losses in objective function (9), only constraint (10) applies.

3.2. Decision Making. A set of nondominated solutions will be obtained after solving the TNEP model. To choose the final plan, a fuzzy satisfying method based on the distance metric method is adopted to help the planner make the decision. The fuzzy sets are defined by a linear membership functions as follows:

$$
\mu_{f_{i}}(X)= \begin{cases}0 & f_{i}(x)>f_{i}^{\max } \\ \frac{f_{i}^{\max }-f_{i}(x)}{f_{i}^{\max }-f_{i}^{\min }} & f_{i}^{\min } \leq f_{i}(x) \leq f_{i}^{\max } \\ 1 & f_{i}(x)<f_{i}^{\min }\end{cases}
$$

The membership value ranges from 0 to 1 , which represents the satisfied degree of membership in a fuzzy set. After assigning membership function to each objective of every solution in the Pareto-optimal front, the decision maker needs to choose the desirable level $\mu_{d i}$ for each objective, and the final solution can be achieved by solving the following optimization problem:

$$
\min _{X \in \text { Solutionset }} \sum_{i=1}^{m}\left|\mu_{d i}-\mu_{f i}(x)\right|^{n}, \quad n \geq 1 .
$$

Since the value of $\left|\mu_{d i}-\mu_{f i}(x)\right|$ ranges from 0 to 1 , so a larger $n$ means the final scheme is less sensitive to the desirable level [7].

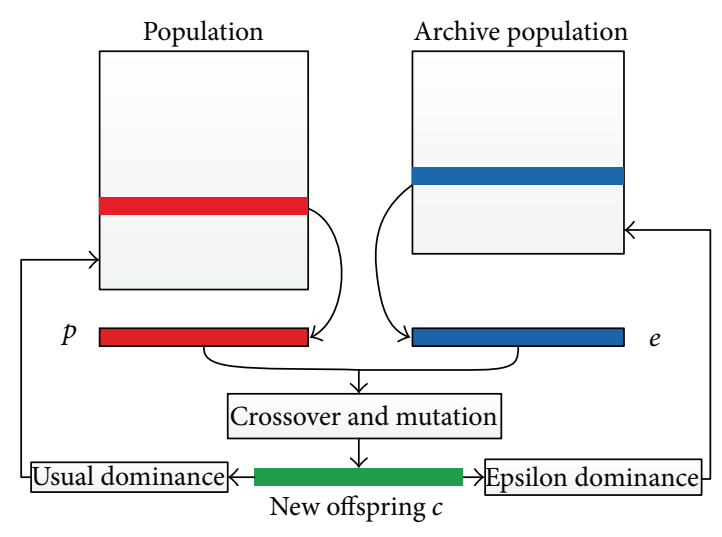

FIgURE 1: $\varepsilon$-MOEA procedure.

\section{Optimization Method}

Most MOEAs developed in the past decade are either good for achieving well-distributed solutions at the expense of a large computational effort or computationally fast at the expense of obtaining a not-so-accurate distribution of solutions [6]. In order to find a good compromise between the diversity of the final solutions and the computational time, here we choose the $\varepsilon$-MOEA to solve the proposed multiobjective optimization problem.

4.1. $\varepsilon$-MOEA Method. $\varepsilon$-MOEA was developed by Deb et al. in 2005 [6] based on the work of Laumanns et al. who first proposed the $\varepsilon$-dominance concept [29]. Like most of the multiobjective evolutionary algorithms, the basic idea of $\varepsilon$-MOEA to obtain the final Pareto-optimal front is an iterative search algorithm through several operations such as reproduction, crossover, and mutation. Figure 1 shows the iterative procedure of $\varepsilon$-MOEA. Firstly, a population $P(0)$ is initialized by a set of solutions which are randomly selected from the feasible solution space, and the fitness value of each solution is evaluated. At the same time, the nondominated solutions from $P(0)$ are used to form an archive population $E(0)$, and the iteration count $t$ is set at zero. Secondly, the solutions $p$ and $e$ are, respectively, selected from the population $P(t)$ and $A(t)$ to create a new solution $c$ through crossover and mutation. Then, the populations $P(t)$ and $A(t)$ are updated by usual dominance criterion and $\varepsilon$ dominance criterion, respectively, using the solution $c$. This iterative search procedure is running until the terminated condition is satisfied. Finally, the obtained archive members are reported as the final solutions.

Different from the existing MOEAS, the $\varepsilon$-MOEA is preferable mainly in two aspects. One is that it uses a steadystate evolutionary algorithm (EA) in which every offspring is compared with the parent population as soon as it is created, rather than a generational evolutionary algorithm in which all $N$ population members (offspring) are created before comparing them with parent solutions. So, the parent population can be updated in a steady-state manner which provides better chances for creating good offspring solutions. The other is that it implements the $\varepsilon$-dominance concept to 


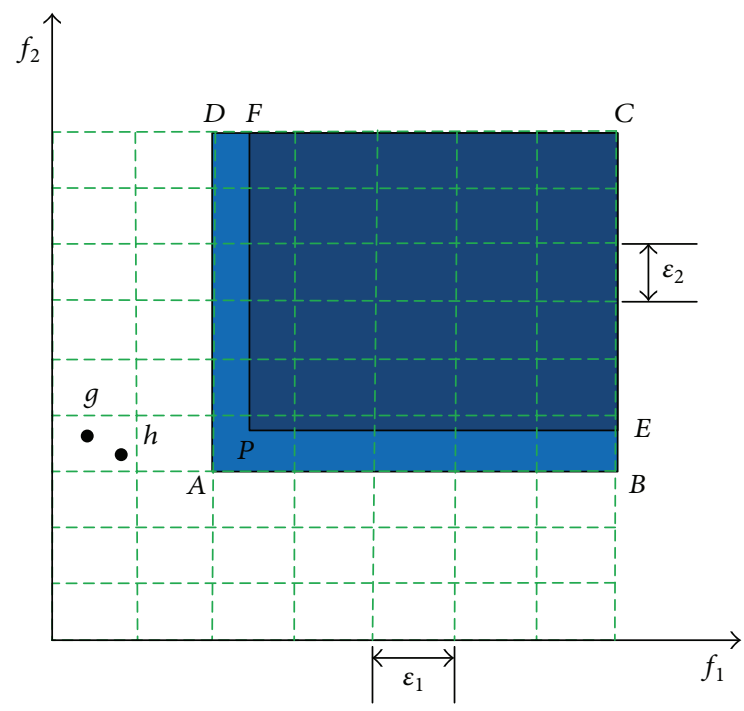

FIgURE 2: The $\varepsilon$-dominance concept.

update the archive population. The difference between the $\varepsilon$ dominance and the usual domination concept is illustrated in Figure 2, in which the whole objective space (2 dimension) is divided into hyperboxes, each having $\varepsilon_{j}$ size in the $j$ th objective, and $\varepsilon_{j}$ represents the allowable tolerance in the $j$ th objective set by user. In Figure 2, it can be seen that the solution $P$ by the usual domination concept only dominates the region PECFP while solution by $\varepsilon$-dominance is the entire region $\mathrm{ABCDA}$. Both solutions are in the minimization sense. It is clear that the $\varepsilon$-dominance criterion does not allow two nondominated solutions with a difference less than $\varepsilon_{i}$ in the $i$ th objective to be both present in the final archive, such as point $g$ and point $h$ in Figure 2, thereby allowing a good diversity to be maintained in the final Pareto-optimal front. Besides, it allows the user to choose a suitable $\varepsilon_{i}$ depending on their desired resolution in the $i$ th objective, so it also has a practical significance.

Because the value of $\varepsilon$ is closely related to the Paretooptimal front of the archive population, and the final Pareto-optimal front of real-world multiobjective problem is unknown, it is hard for the user to choose an appropriate value of $\varepsilon$. In this paper, we adopt a self-adaption strategy for the parameter $\varepsilon$ [30] to solve this problem. It is described in the following equation:

$$
\varepsilon=\varepsilon_{0}\left(\frac{P}{P^{\prime}}\right)^{1 /(m-1)},
$$

where $\varepsilon_{0}$ is a predefined value by the user, $m$ is the total number of objectives, and $P$ and $P^{\prime}$ are the current and expected number of the nondominated solutions in the archive population, respectively. By applying the selfadaption $\varepsilon$ in the $\varepsilon$-MOEA, we could achieve the expected number of nondominated solutions approximately with a well distributed front.

Another performance improvement technique of the original $\varepsilon$-MOEA in this paper is the use of self-adaptive probabilities of crossover and mutation to achieve the

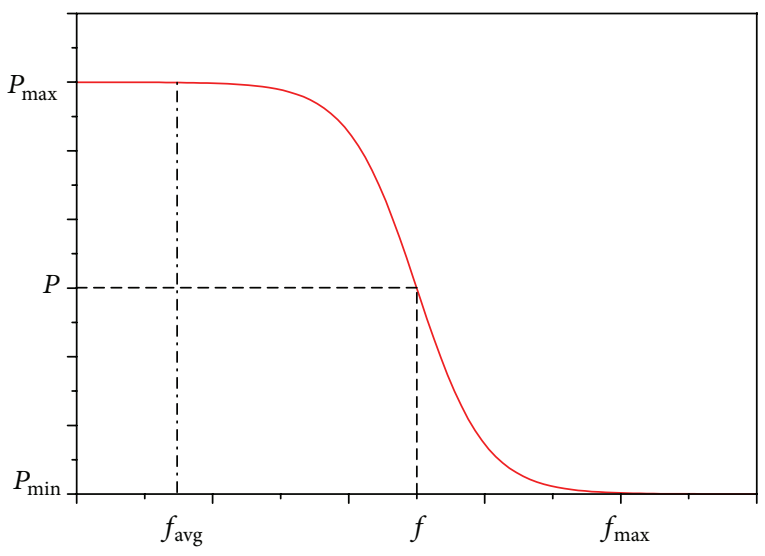

Figure 3: The adaptive adjusting curve of $P_{c}$ and $P_{m}$.

twin goals of maintaining diversity among populations and strengthening the convergence capacity of algorithm. The expressions for crossover and mutation take forms as follows [31]:

$$
P_{c}=\left\{\begin{array}{lr}
\left(P_{c \max }-P_{c \min }\right) & \\
\times\left(1+\exp \left(9.903438\left(\frac{2\left(f-f_{\mathrm{avg}}\right)}{\left(f_{\max }-f_{\text {avg }}\right)}-1\right)\right)\right)^{-1} & \\
+P_{c \min } & f \geq f_{\text {avg }} \\
P_{c \min } & f<f_{\text {avg }}
\end{array}\right.
$$

$$
P_{m}=\left\{\begin{array}{lr}
\left(P_{m \text { max }}-P_{m \text { min }}\right) & \\
\times\left(1+\exp \left(9.903438\left(\frac{2\left(f-f_{\text {avg }}\right)}{\left(f_{\text {max }}-f_{\text {avg }}\right)}-1\right)\right)\right)^{-1} & \\
+P_{m \text { min }} & f \geq f_{\text {avg }} \\
P_{m \text { min }} & f<f_{\text {avg }}
\end{array}\right.
$$

$$
f=-\left(f_{1}+1000 f_{2}+f_{3}\right),
$$

where $P_{c \max }, P_{c \text { min }}$ are the predefined maximum and minimum probabilities of crossover. $P_{m \text { max }}, P_{m \text { min }}$ are the predefined maximum and minimum probabilities of mutation. The fitness value $f$ in (15) is the biggest of the selected parent individuals, such as $p$ and $e$ in Figure 1, which can be obtained from (17). While the $f$ in (16) is the fitness value of the individual chosen to mutate. $f_{1}, f_{2}$, and $f_{3}$ are the objective function values mentioned before. $f_{\text {avg }}$ is the average fitness value of the archive population. The dynamic change of $P_{c}$ and $P_{m}$ is illustrated by Figure 3, in which $P_{c \text { max }}$ and $P_{m \text { max }}$ are represented by $P_{\max } ; P_{\min }$ represents $P_{c \text { min }}$ and $P_{m \text { min }} ; P$ represents the current probabilities of crossover and mutation. Since the variation of probabilities of crossover and mutation depends on the fitness value of the solutions, the solutions with high fitness value are protected, and the solutions with subaverage fitness are 
Journal of Applied Mathematics

disrupted to reproduce good ones. Thereby, the self-adaptive probabilities of crossover and mutation will guarantee the modified $\varepsilon$-MOEA to converge to the global optimum in fewer generations and get stuck at a local optimum fewer times than the original one with constant probabilities of crossover and mutation.

4.2. Proposed Algorithm. Figure 4 shows the flowchart of the proposed algorithm for the multiobjective TNEP problem. The algorithm begins with the initialization of the population $P(0)$, in which the candidate lines of each solution coded by binary variable are generated by random number. Then, using the PPF based on the extended $2 m+1$ point-estimate method, the cost of network losses and the amount of line overload capacity under normal operation as well as single contingency conditions are evaluated. Having the investment cost calculated by (5), the initial archive population $E(0)$ can be obtained by the nondominated solutions from the $P(0)$. After the initialization of $P(0)$ and $E(0)$, the algorithm starts to perform iterative search for the final optimal front, the procedure is the same as mentioned in part A, the difference is the self-adaptive probabilities of crossover, and mutations need to be updated based on the fitness value of the solutions. Besides, when using the new solution to update the archive population, if the amount of line overload capacity of new solution under normal and single contingency condition exceeds the $20 \%$ of total load capacity, it will be discarded directly without the judgment of the $\varepsilon$-dominance concept Because the security criteria of the solution are not satisfied in the practical engineering, it will not be chosen by the planner in the final decision making stage. This searching process is iterated until the termination criterion is satisfied, and the final archive members are reported as the obtained solutions. Upon the final optimal front obtained from the modified $\varepsilon$-MOEA, finally, the fuzzy satisfying method is implemented to select the final optimal solution based on the preference of the planner.

\section{Case Study}

5.1. IEEE 24-Bus Test System. In this section, a modified IEEE 24-bus test system is used to demonstrate the performance of the proposed algorithm. Assume that the system will be expanded for future conditions with the generation and load demand increasing by 2.2 times their original values, that is, load level of $6720 \mathrm{MW}$ and generation level of $7490 \mathrm{MW}$. This system has 32 generators, 38 existing lines, and 89 candidate lines (including substations). The candidate lines which can be added in 29 existing right-of-ways and ten new right-ofways are limited to three and two, respectively; up to four transformers can be installed in the substations; all data of the candidate lines can be found in [7]. Unit electricity price and the annual network losses hours as well as discount rate and period are based on [28].

The test system is divided into two regions, namely, the northern region with nodes $1-10$ and the southern region with nodes 11-24, and three wind farms are integrated to each region. In order to investigate the effects of the wind power on the test system, five different cases are designed.
TABLE 1: Wind power input data of test system.

\begin{tabular}{lcccc}
\hline $\begin{array}{l}\text { Wind farm } \\
\text { integration node }\end{array}$ & $\begin{array}{c}\text { Mean } \\
(\mathrm{MW})\end{array}$ & $\begin{array}{c}\text { Standard } \\
\text { deviation } \\
(\mathrm{MW})\end{array}$ & Skewness & Kurtosis \\
\hline 1 & 103.4 & 66 & 0.9269 & 3.6858 \\
2 & 100.7 & 63.2 & 1.0265 & 3.5026 \\
7 & 102.1 & 65.5 & 0.9604 & 3.2001 \\
15 & 101.7 & 71.7 & 1.0385 & 3.2713 \\
18 & 96.1 & 69.6 & 1.1253 & 4.2175 \\
23 & 102.3 & 82.7 & 1.4435 & 4.4517 \\
\hline
\end{tabular}

Case 1. The test system is only supplied by the conventional units.

Case 2. Partial conventional units are replaced by wind energy, and the total wind energy penetration is $5 \%$ of all load capacities for the years to come, and the outputs of wind farms are independent.

Case 3. The correlation among wind farms is considered; other conditions are the same as Case 2.

Case 4. The total wind energy penetration is $10 \%$; other conditions are the same as Case 2.

Case 5. The total wind energy penetration is $15 \%$; other conditions are the same as Case 3.

In Case 2, conventional units in nodes 1, 2, 7, 15, 18, and 23 are replaced by wind turbines, and input data of six wind farms are shown in Table 1. In Case 3, wind farms within the same region are correlated with a correlation coefficient 0.8 ; the correlation coefficient between two regions is 0.1 . The wind energy penetration of Case 4 is 1.5 times of Case 2, so the mean value and standard deviation of wind farms in Case 4 will be increased by 1.5 times the value in Table 1, and the skewness and kurtosis remain the same.

From Table 1, it can be seen that the input data of wind farms are just the first four statistical moments of wind power. Since the input information of wind power is a large number of discreet data and the probability distribution function is hard to obtain, the relatively simple input data will provide convenience molding wind power.

Figure 5(a) shows the final results of Cases 2 and 3 achieved by the proposed method. The optimal-Pareto front of Case 2 is located in front of the optimal front of Case 3 , and this spatial relationship in three-dimensional space is more clearly reflected in Figure 5(b). Such relationship indicates that, as the correlation between wind farms is considered, the intermittency and volatility of wind power will increase, so more investment cost is required to reach the balance between economic and security criteria of power system.

Final results of Cases 4 and 5 are presented in Figure 6, and the same conclusion can be obtained as in Figure 5. However, it can be noticed that the distance between the Pareto-optimal front of Cases 4 and 5 is wider than that between Cases 2 and 3; the reason is that with the increasing 


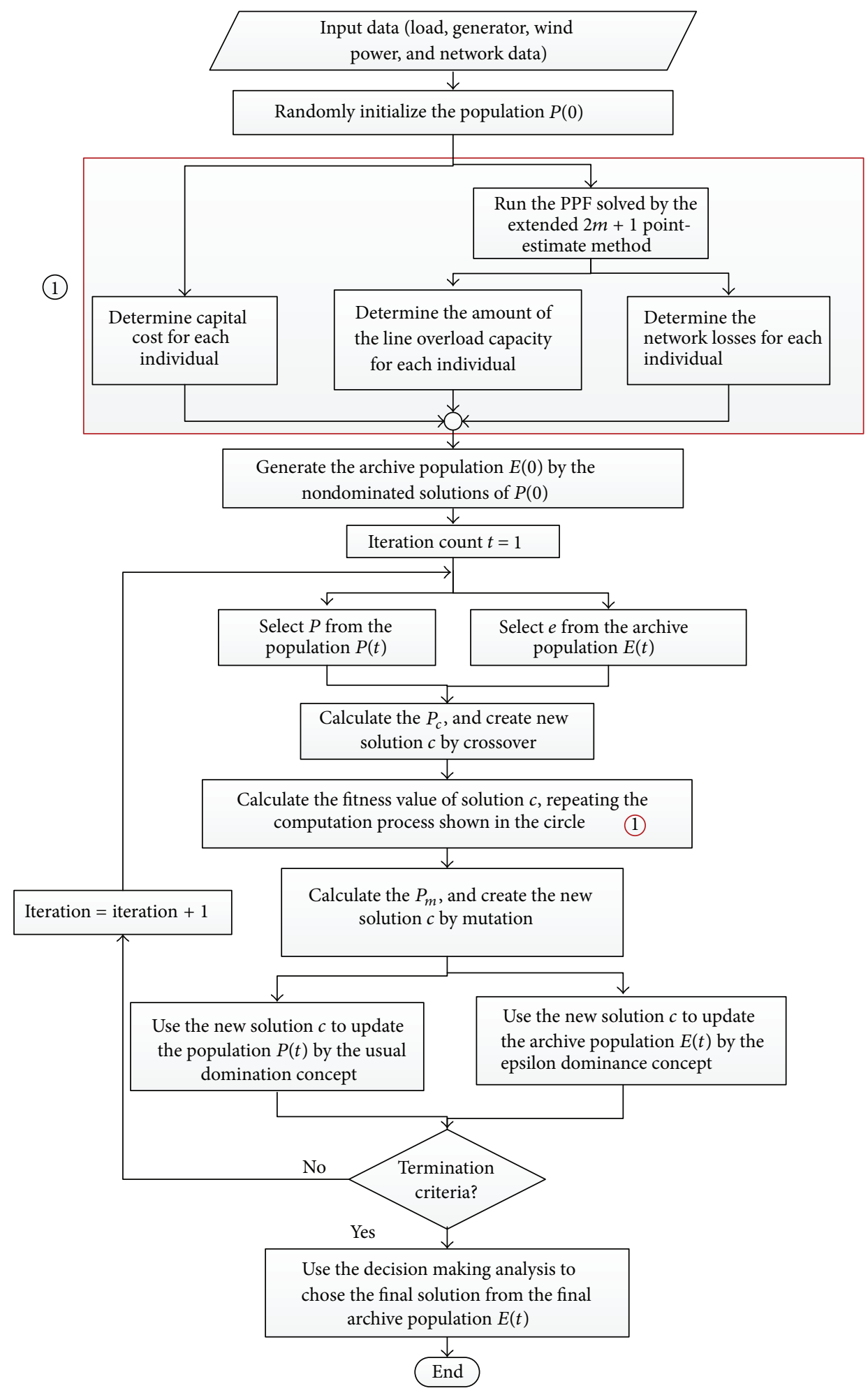

FIGURE 4: Flowchart of the proposed algorithm.

penetration of wind power, the influence of the correlation between wind farms will be more significant on the transmission network.

Since the final results of the proposed method are a set of nondominated solutions, the decision making analysis method needs to be applied to obtain the final optimal plan. Table 2 shows the final scheme of Cases 1, 3, and 5 obtained by the fuzzy satisfying method, when the desirable level for each objective set by planer is $0.6,1$, and 0.7 , respectively. As can be seen from Table 2, compared to Cases 3 and 5, due to 


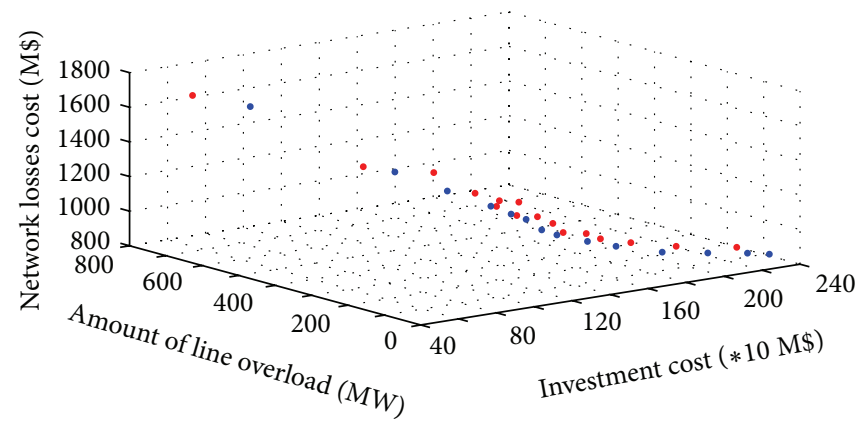

- Case 3

- Case 2

(a) Final Pareto front of Cases 2 and 3

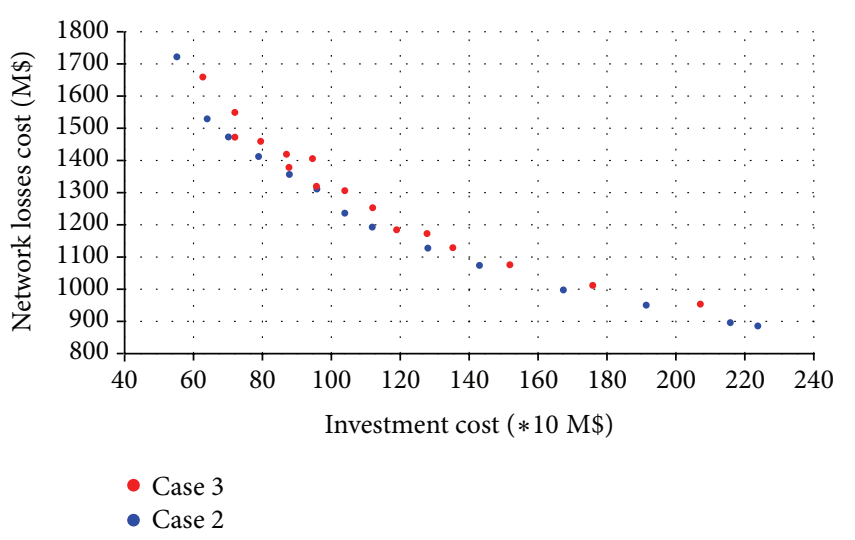

(b) Trade-off between investment cost and network losses cost in Cases 2 and 3

Figure 5: Final results of Cases 2 and 3.

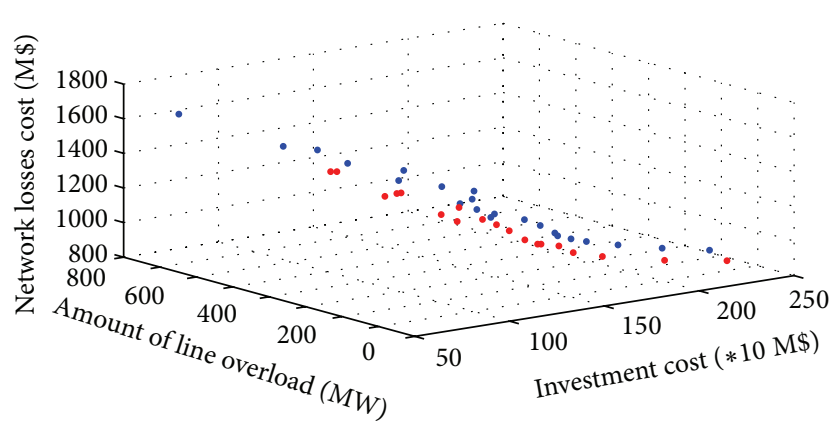

- Case 5

- Case 4

(a) Final Pareto front of Cases 4 and 5

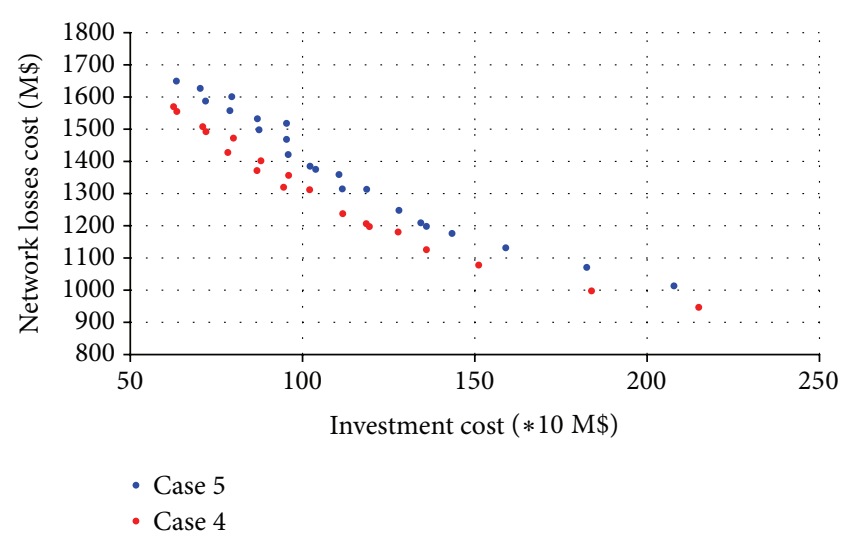

(b) Trade-off between investment cost and network losses cost in Cases 4 and 5

FIGURE 6: Final results of Cases 4 and 5.

TABle 2: Final plan of Cases 1, 3, and 5.

\begin{tabular}{lccc}
\hline Scheme & $\begin{array}{c}\text { Investment cost } \\
(\mathrm{M} \$)\end{array}$ & $\begin{array}{c}\text { Amount of line } \\
\text { overload }(\mathrm{MW})\end{array}$ & $\begin{array}{c}\text { Network losses } \\
\text { cost }(\mathrm{M} \$)\end{array}$ \\
\hline Case 1 & 940.7418 & 2.55659 & 1235.44 \\
Case 3 & 1189.26 & 20.0876 & 1184.35 \\
Case 5 & 1279.66 & 21.5414 & 1247.85 \\
\hline
\end{tabular}

the absence of wind power in Case 1 , the security criterion of transmission network, which is reflected by the amount of line overload, can still be guaranteed with less investment. And as the figure of Cases 3 and 5 shows, with the increasing integration of wind power, more investment is required to reach the same security criterion. This conclusion can also be drawn in Figure 7, that the final Pareto-optimal front of Cases 1,3 , and 5 in the trade-off graph is clearly ranked one by one with the increasing integration of wind power. Figure 8 shows the final planning result of Case 5 , in which transmission lines around wind farms and important loads are strengthened.
5.2. Real Test System. The proposed method is applied to a $220 \mathrm{kV}$ practical power system in the south of China as well. This system has 233 buses, 452 existing lines, and 164 candidate lines. It is assumed that five years later the total installed capacity will be $4729 \mathrm{MW}$ and the total load is $4158 \mathrm{MW}$. There are four wind farms integrated into the system, including three wind farms with correlation coefficient 0.6 in the west and one in the northeastern, and the correlation between wind farms within two regions is 0.1 ; the total penetration of wind farm is $15 \%$ of the installed capacity. Table 3 presents the input data of the four wind farms. Unit electricity price, the annual network losses hours, discount rate, and period also adopt values in part A.

Two models, one considering the correlation between wind farms while the other not, are solved by the proposed method, and the final Pareto-optimal front in twodimensional space is shown in Figure 9. As can be seen from Figure 9(a), while at the same level of line overload, the investment of the second model is smaller than that of the first model considering wind power correlation. However, 


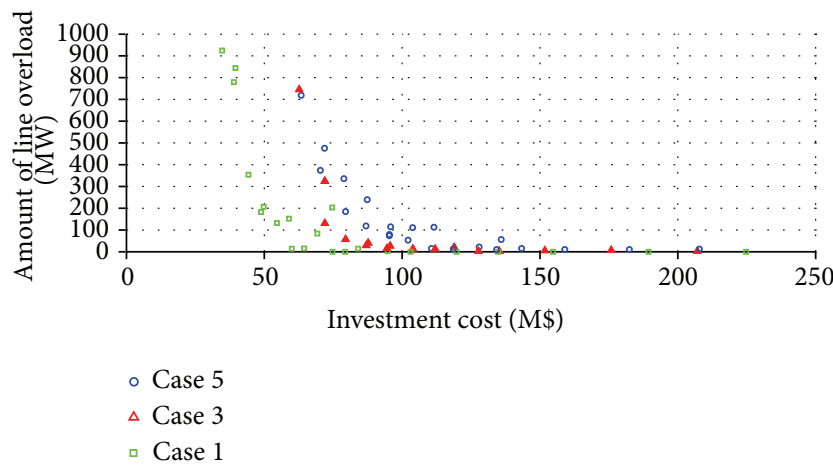

(a) Trade-off between investment cost and the amount of line overload of Cases 1,3 , and 5

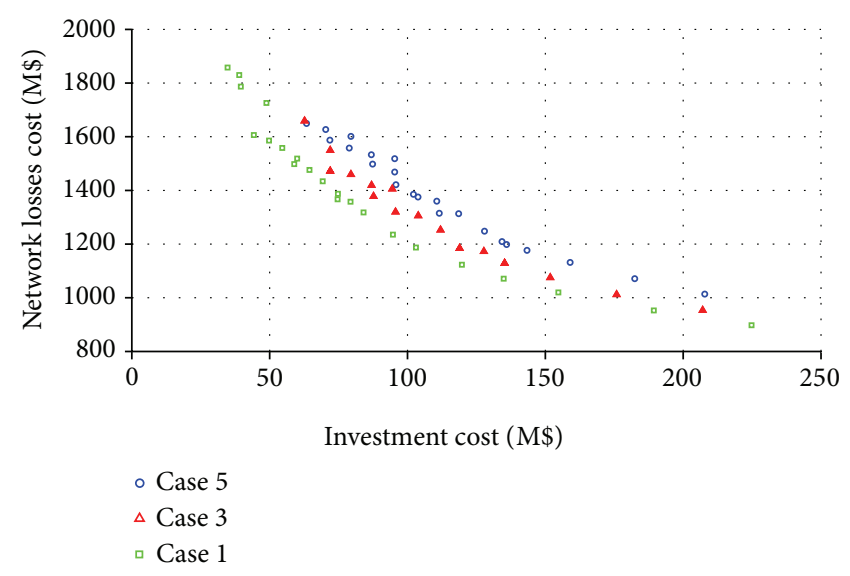

(b) Trade-off between investment cost and network losses of Cases 1,3, and 5

Figure 7: Trade-off graphs of Cases 1, 3, and 5.

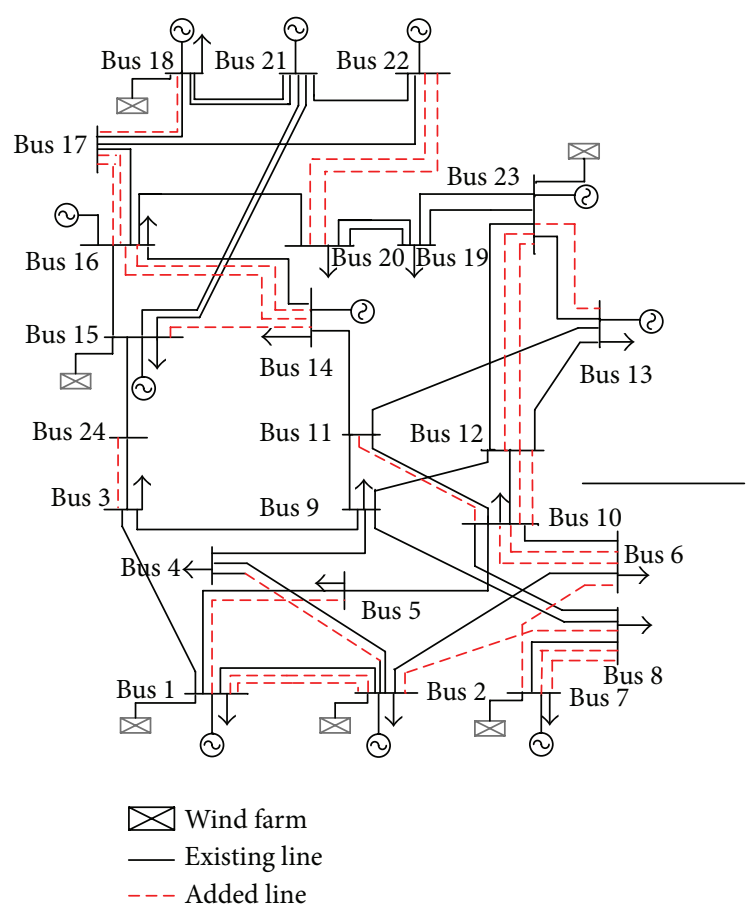

Figure 8: Final plan of Case 5.

the consideration of wind power correlation leads to a less ideal but more realistic situation, and the result achieved by model 1 is more reliable compared to model 2. Besides, in Figures 9(a) and 9(b), there are five points concentrating in the lower right corner with a clear distance away from the others. These five points represent planning schemes in which several expensive long distance lines are chosen. Though the investments are large, these lines can tremendously change the original transmission structure, and the distribution of power flow can be well improved, so the network loss costs of these five solutions are relatively low.
TABLE 3: Wind power input data of practical system.

\begin{tabular}{lcccc}
\hline Wind farm & $\begin{array}{c}\text { Mean } \\
(\mathrm{MW})\end{array}$ & $\begin{array}{c}\text { Standard } \\
\text { deviation } \\
(\mathrm{MW})\end{array}$ & Skewness & Kurtosis \\
\hline 1 & 200.01 & 165.33 & 1.243 & 3.674 \\
2 & 165.37 & 60.73 & 1.308 & 3.970 \\
3 & 300.75 & 123.8 & 0.868 & 2.494 \\
4 & 63.47 & 52.78 & 1.155 & 3.198 \\
\hline
\end{tabular}

TABLE 4: Final planning scheme of the real test system.

\begin{tabular}{cccccc}
\hline Desirable level & $\begin{array}{c}\text { Investment } \\
\text { cost (M\$) }\end{array}$ & $\begin{array}{c}\text { Amount of } \\
\text { line } \\
\text { overload } \\
(\mathrm{MW})\end{array}$ & $\begin{array}{c}\text { Network } \\
\text { losses cost } \\
(\mathrm{M} \$)\end{array}$ \\
\hline 0.2 & 1 & 1 & 18390.9 & 30.7138 & 1663.62 \\
0.4 & 1 & 1 & 17740.4 & 68.3243 & 1719.06 \\
0.6 & 1 & 1 & 12395 & 33.4318 & 1956.2 \\
\hline
\end{tabular}

Assuming the planner has high requirements on the security of transmission system and wants to minimize the network losses cost, Table 4 shows the final plan of the accurate model under different desirable level for investment.

Figure 10 shows the final planning scheme in Table 4 when the desirable level for investment is 0.2 . As can be seen from Figure 10, transmission lines around wind farms 1,2 , and 3 are strengthened to improve the security of transmission system. Since the output power of wind farm 4 is small and the existing lines satisfied the security requirement, there are no new added lines around wind farm 4. Besides, because two expensive long distance lines are added, the distribution of power flow is well improved and the network losses cost is relatively low. 


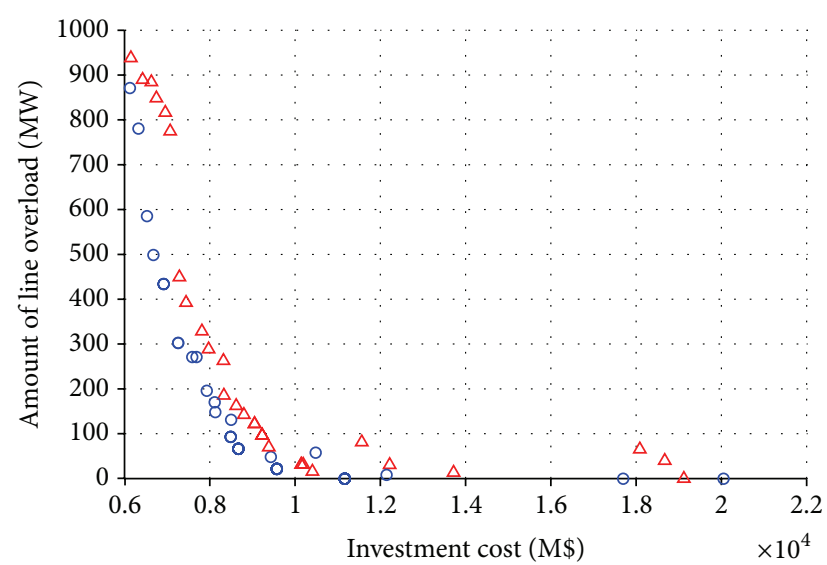

- Model without correlation

$\triangle$ Model with correlation

(a) Trade-off between investment cost and the amount of line overload

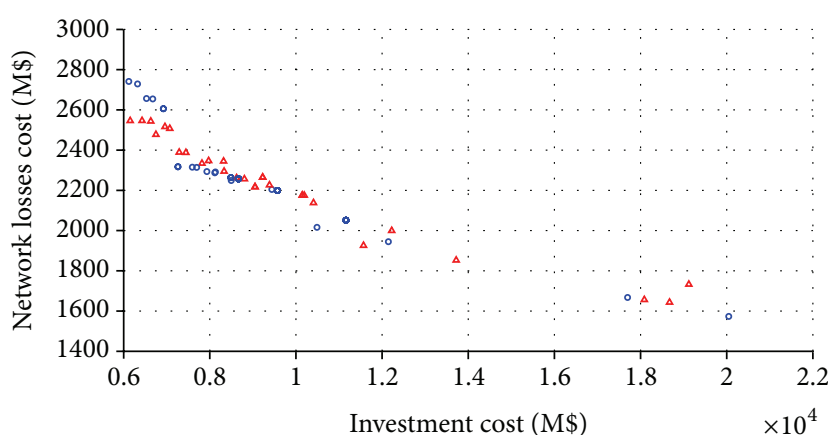

- Model without correlation

$\Delta$ Model with correlation

(b) Trade-off between investment cost and network losses

FIGURE 9: Trade-off graphs of practical power system.

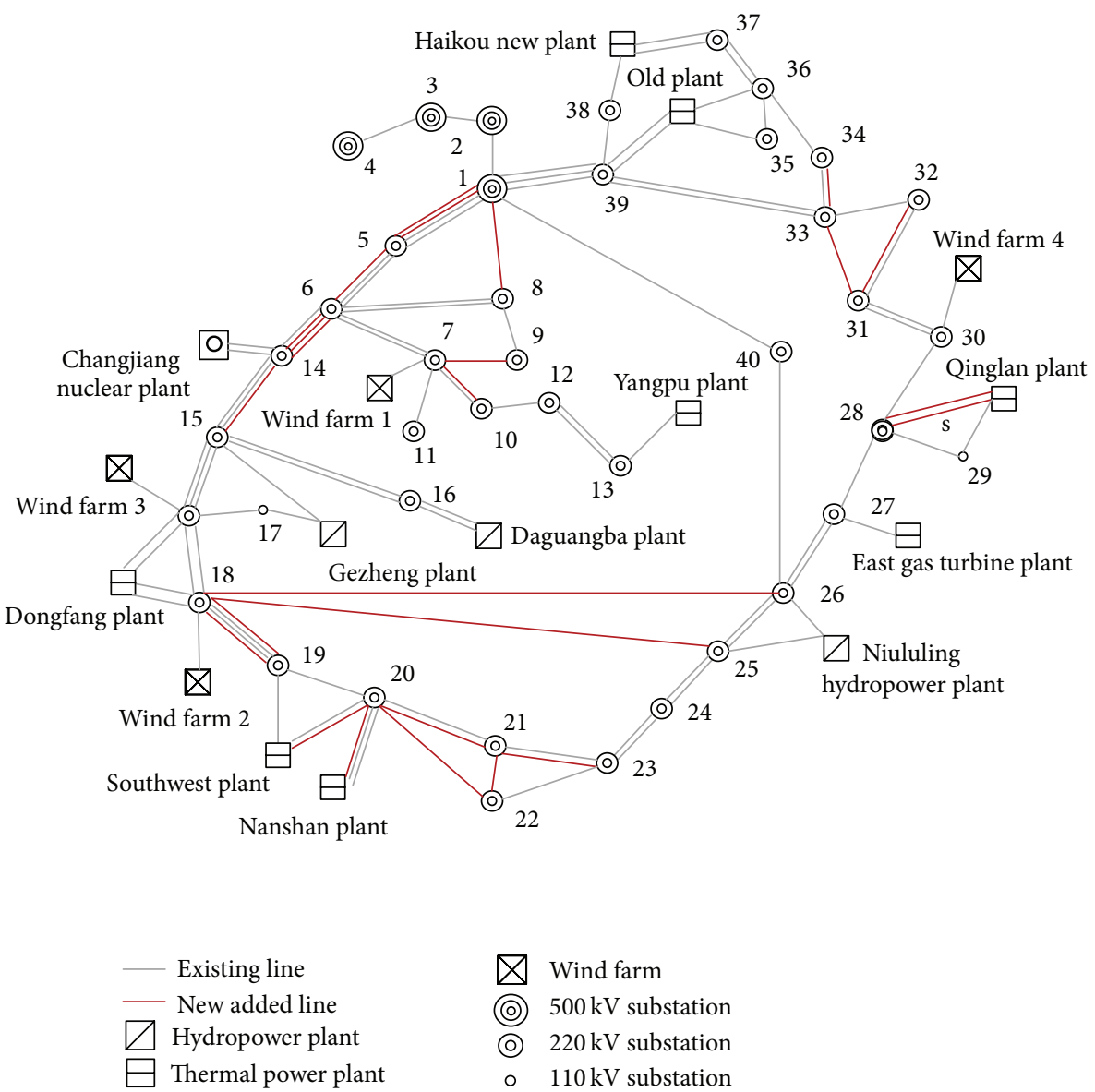

FIGURE 10: Final planning scheme of real power system. 


\section{Conclusion}

This paper established a multiobjective TNEP model, in which the investment cost and network losses cost as well as the line overload capacity both under the normal operation and single contingency condition are considered. To handle the uncertainty and correlation of wind power in TNEP model, an extended $2 m+1$ point-estimate method is applied to transform the uncertain model into a solvable deterministic model. Then, a modified $\varepsilon$-MOEA and a fuzzy satisfying method are used to achieve the final planning scheme. This method has the following advantages.

(1) The input data is very simple, because only the first four statistical moments and correlation coefficients of output power of wind farms are required.

(2) The model is more precise by considering the correlation between wind farms, and it has been demonstrated by the final results of IEEE 24 and real power system.

(3) Since the final results are a set of nondominated solutions, planners can flexibly choose the final planning scheme according to the practical situation.

Because of considering the $\mathrm{N}-1$ check in the objective function, the evaluation of objective function is timeconsuming. In our next work, the proposed method will be further improved by the application of parallel computation method.

\section{Conflict of Interests}

The authors declare that there is no conflict of interests regarding the publication of this paper.

\section{Acknowledgment}

This work was supported in part by the National High Technology Research and Development Program of China (863 Program) under Grant 2012AA050201.

\section{References}

[1] H. Sun and D. C. Yu, "A multiple-objective optimization model of transmission enhancement planning for independent transmission company (ITC)," in Proceedings of the 2000 Power Engineering Society Summer Meeting, pp. 2033-2038, usa, July 2000.

[2] T. S. Chung, K. K. Li, G. J. Chen, J. D. Xie, and G. Q. Tang, "Multi-objective transmission network planning by a hybrid GA approach with fuzzy decision analysis," International Journal of Electrical Power and Energy Systems, vol. 25, no. 3, pp. 187-192, 2003.

[3] Z. Xu, Z. D. Dong, and K. P. Wong, "A hybrid planning method for transmission networks in a deregulated environment," IEEE Transactions on Power Systems, vol. 21, no. 2, pp. 925-932, 2006.

[4] E. Zitzler and L. Thiele, "Multiobjective evolutionary algorithms: A comparative case study and the strength Pareto approach," IEEE Transactions on Evolutionary Computation, vol. 3, no. 4, pp. 257-271, 1999.
[5] K. Deb, A. Pratap, S. Agarwal, and T. Meyarivan, "A fast and elitist multiobjective genetic algorithm: NSGA-II," IEEE Transactions on Evolutionary Computation, vol. 6, no. 2, pp. 182197, 2002.

[6] K. Deb, M. Mohan, and S. Mishra, "Evaluating the $\varepsilon$-domination based multi-objective evolutionary algorithm for a quick computation of Pareto-optimal solutions," Evolutionary Computation, vol. 13, no. 4, pp. 501-525, 2005.

[7] P. Maghouli, S. H. Hosseini, M. O. Buygi, and M. Shahidehpour, "A multi-objective framework for transmission expansion planning in deregulated environments," IEEE Transactions on Power Systems, vol. 24, no. 2, pp. 1051-1061, 2009.

[8] P. Maghouli, S. H. Hosseini, M. Oloomi Buygi, and M. Shahidehpour, "A scenario-based multi-objective model for multi-stage transmission expansion planning," IEEE Transactions on Power Systems, vol. 26, no. 1, pp. 470-478, 2011.

[9] M. Moeini-Aghtaie, A. Abbaspour, and M. Fotuhi-Firuzabad, "Incorporating large-scale distant wind farms in probabilistic transmission expansion planning-part I: theory and algorithm," IEEE Transactions on Power Systems, vol. 27, no. 3, pp. 1585-1593, 2012.

[10] Y. Wang, H. Cheng, C. Wang et al., "Pareto optimality-based multi-objective transmission planning considering transmission congestion," Electric Power Systems Research, vol. 78, no. 9, pp. 1619-1626, 2008.

[11] W. F. Esser, P. Ghose, and K. Chen, "Decision analysis for electric power systems engineering and management," IEEE Transactions on Power Apparatus and Systems, vol. 96, no. 2, pp. 447-456, 1977.

[12] C. Chompoo-Inwai, L. Wei-Jen, P. Fuangfoo, M. Williams, and J. R. Liao, "System impact study for the interconnection of wind generation and utility system," IEEE Transactions on Industry Applications, vol. 41, no. 1, pp. 163-168, 2005.

[13] X. Cui, W. Li, X. Ren, F. Xue, and Y. Fang, "Review of transmission planning with large-scale wind power integration," in Proceedings of the Asia-Pacific Symposium on Electromagnetic Compatibility (APEMC '12), pp. 285-288, Singapore, May 2012.

[14] H. Yu, C. Y. Chung, K. P. Wong, and J. H. Zhang, "A chance constrained transmission network expansion planning method with consideration of load and wind farm uncertainties," IEEE Transactions on Power Systems, vol. 24, no. 3, pp. 1568-1576, 2009.

[15] G. A. Orfanos, P. S. Georgilakis, and N. D. Hatziargyriou, "Transmission Expansion Planning of Systems With Increasing Wind Power Integration," IEEE Transactions on Power Systems, 2012.

[16] A. M. Leite da Silva, S. M. P. Ribeiro, V. L. Arienti, R. N. Allan, and M. B. do Coutto, "Probabilistic load flow techniques applied to power system expansion planning," IEEE Transactions on Power Systems, vol. 5, no. 4, pp. 1047-1053, 1990.

[17] A. M. Leite Da Silva, L. Manso, W. D. S. Sales, S. A. Flavio, G. J. Anders, and L. C. de Resende, "Chronological power flow for planning transmission systems considering intermittent sources," IEEE Transactions on Power Systems, vol. 27, no. 4, pp. 2314-2322, 2012.

[18] J. M. Morales and J. Pérez-Ruiz, "Point estimate schemes to solve the probabilistic power flow," IEEE Transactions on Power Systems, vol. 22, no. 4, pp. 1594-1601, 2007.

[19] P. Sánchez Martín, A. Ramos, and J. F. Alonso, "Probabilistic midterm transmission planning in a liberalized market," IEEE Transactions on Power Systems, vol. 20, no. 4, pp. 2135-2142, 2005. 
[20] X. Ai, J. Wen, T. Wu, S. Sun, and G. Li, "A practical algorithm based on point estimate method and Gram-Charlier expansion for probabilistic load flow calculation of power systems incorporating wind power," in Proceedings of the CSEE, pp. 16-23, 2013.

[21] H. P. Hong, "An efficient point estimate method for probabilistic analysis," Reliability Engineering \& System Safety, vol. 59, pp. 261-267, 1998.

[22] P. Karki, P. Hu, and R. Billinton, "A simplified wind power generation model for reliability evaluation," IEEE Transactions on Energ Conversion, vol. 21, no. 5, pp. 533-540, 2006.

[23] I. Abouzahr and R. Ramakumar, "An approach to assess the performance of utility-interactive wind electric conversion systems," IEEE Transactions on Energy Conversion, vol. 6, no. 2, pp. 627-638, 1991.

[24] W. Li, Risk Assessment of Power Systems: Models, Methods, and Applications, John Wiley \& Sons, New York, NY, USA, 2005.

[25] T. Niknam, F. Golestaneh, and A. Malekpour, "Probabilistic energy and operation management of a microgrid containing wind/photovoltaic/fuel cell generation and energy storage devices based on point estimate method and self-adaptive gravitational search algorithm," Energy, vol. 43, no. 1, pp. 427437, 2012.

[26] R. Azizipanah-Abarghooee, T. Niknam, A. Roosta, A. R. Malekpour, and M. Zare, "Probabilistic multiobjective windthermal economic emission dispatch based on point estimated method," Energy, vol. 37, pp. 322-335, 2012.

[27] J. M. Morales, L. Baringo, A. J. Conejo, and R. Minguez, "Probabilistic power flow with correlated wind sources," IET Generation, Transmission\& Distribution, vol. 4, no. 5, pp. 641651, 2010.

[28] H. Wei, H. Zijun, C. Junzhao, and Z. Li, “Transmission network planning with $\mathrm{N}-1$ security criterion based on improved multi-objective genetic algorithm," in 2011 4th International Conference on Electric Utility Deregulation and Restructuring and Power Technologies, DRPT 2011, pp. 1250-1254, chn, July 2011.

[29] M. Laumanns, L. Thiele, K. Deb, and E. Zitzler, "Combining convergence and diversity in evolutionary multiobjective optimization," Evolutionary Computation, vol. 10, no. 3, pp. 263-282, 2002.

[30] M. Zhang, W. Luo, X. Pei, and X. Wang, "The self-adaption strategy for parameter $\varepsilon$ in $\varepsilon$-MOEA," in 2008 IEEE Congress on Evolutionary Computation, CEC 2008, pp. 2940-2947, chn, June 2008.

[31] J. Jing and Su. Yong, "An improved adaptive genetic algorithm," Computer Engineering and Applications, pp. 64-69, 2005. 


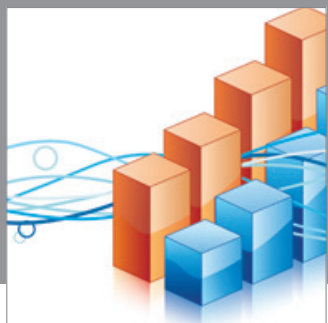

Advances in

Operations Research

mansans

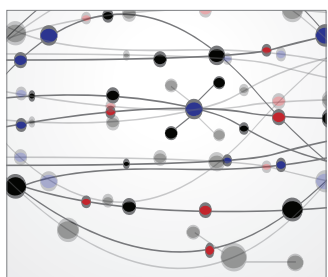

The Scientific World Journal
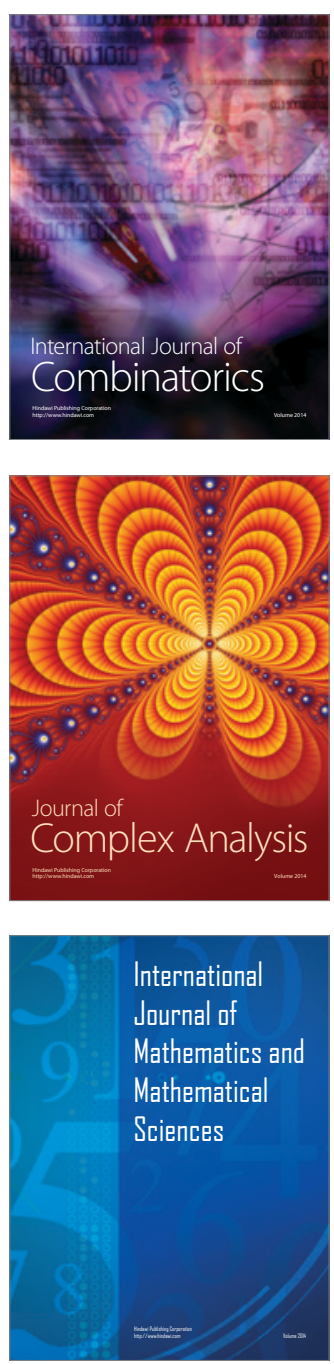
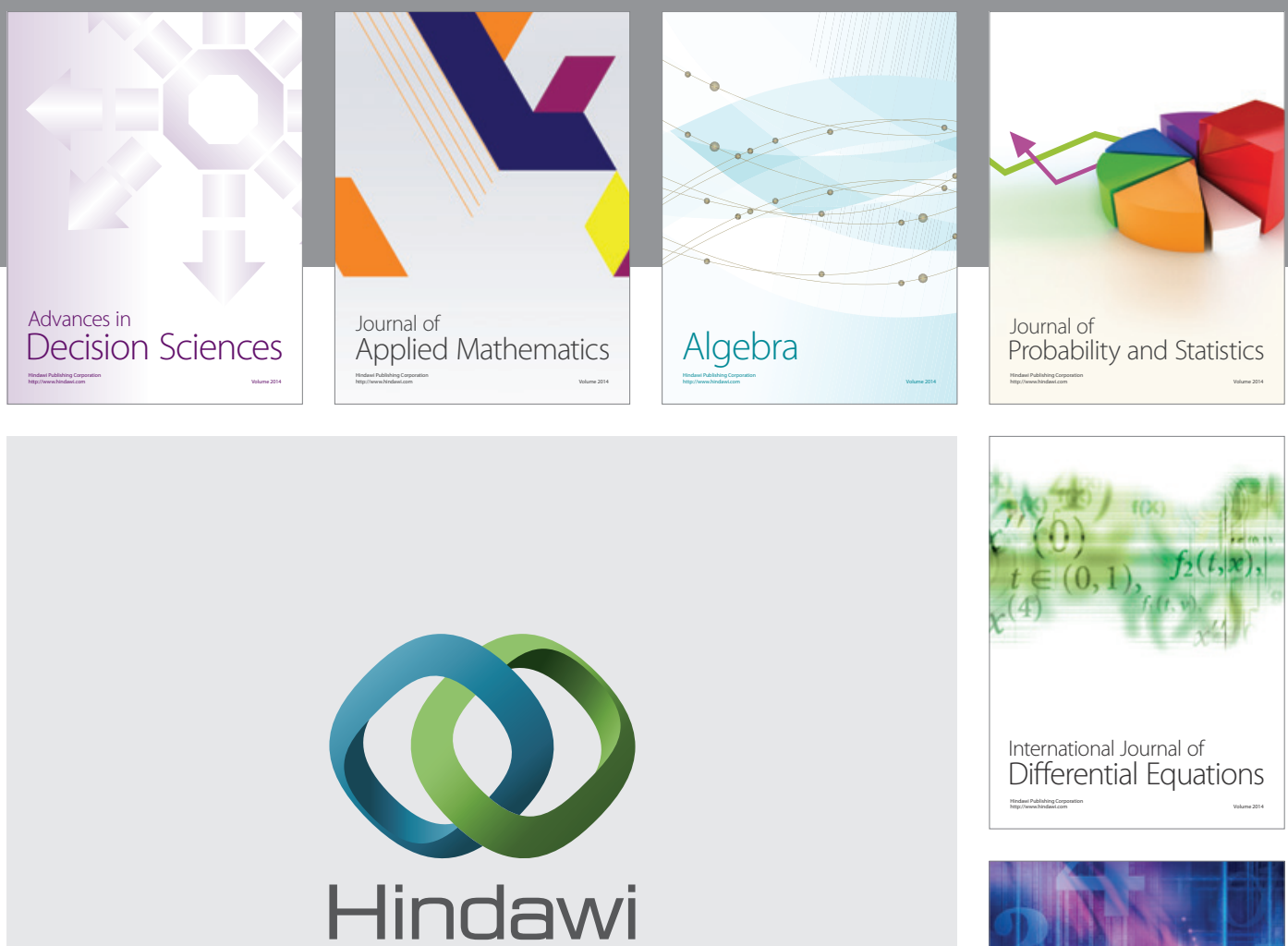

Submit your manuscripts at http://www.hindawi.com
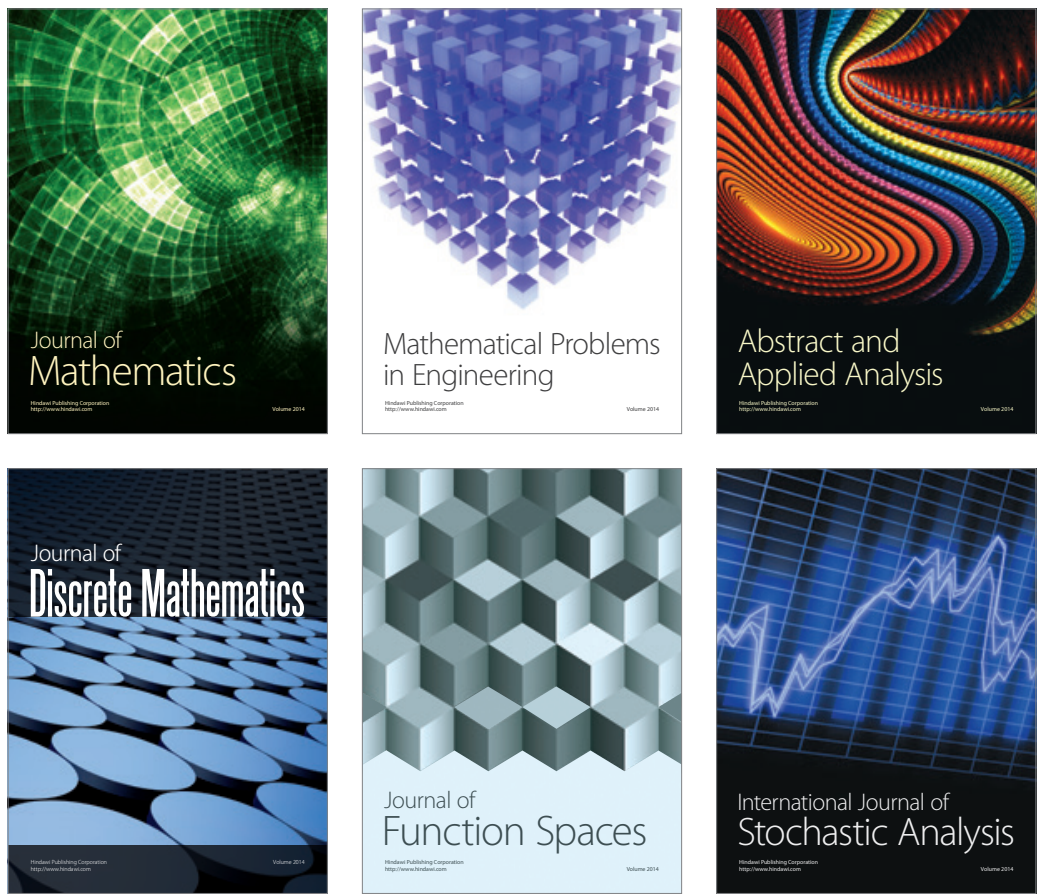

Journal of

Function Spaces

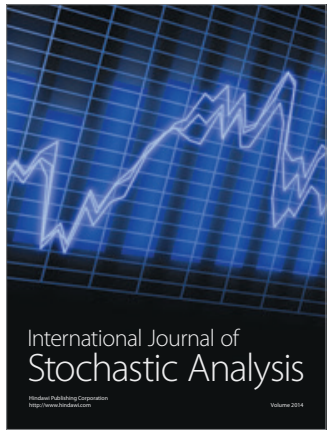

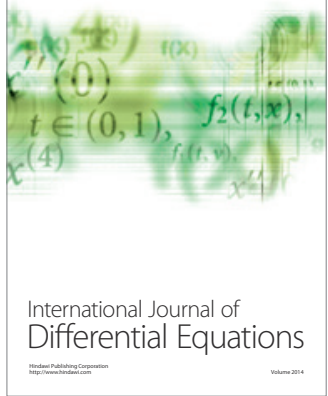
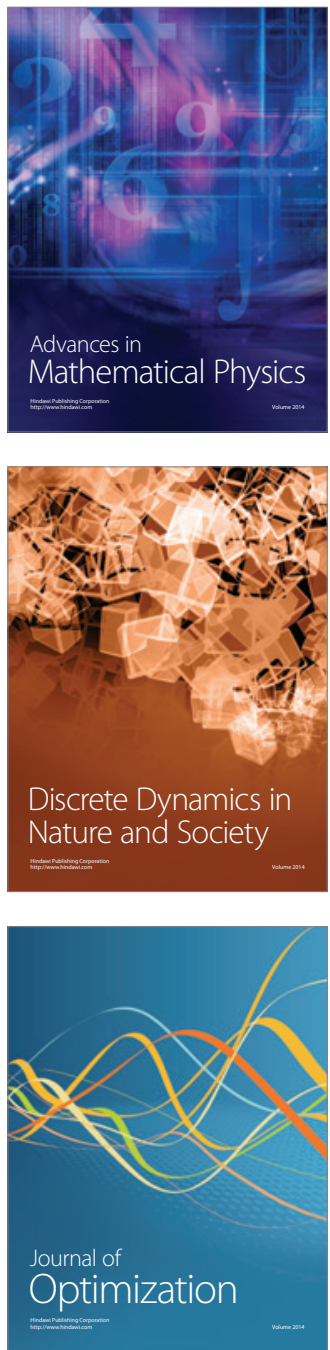